\title{
The Vascular Endothelial Growth Factor's Expression in Gastric Cancer and Progress in Prognostic Research
}

\author{
Li Hongyan ${ }^{1}$, Zhang Shuli ${ }^{2}$, Hou Jianzhang ${ }^{3,}$ * \\ ${ }^{1}$ Department of Medical Technology, Cangzhou Medical College, Cangzhou, China \\ ${ }^{2}$ Obstetrics Ward Five, Yihe District Affiliated with Cangzhou People's Hospital, Cangzhou, China \\ ${ }^{3}$ Department of Hepatobiliary and Pancreatic (Minimally Invasive) Surgery, Cangzhou People's Hospital, Cangzhou, China
}

Email address:

570196466@qq.com (Hou Jianzhang)

${ }^{*}$ Corresponding author

\section{To cite this article:}

Li Hongyan, Zhang Shuli, Hou Jianzhang. The Vascular Endothelial Growth Factor's Expression in Gastric Cancer and Progress in Prognostic Research. American Journal of Biomedical and Life Sciences. Vol. 9, No. 5, 2021, pp. 239-247. doi: 10.11648/j.ajbls.20210905.13

Received: August 30, 2021; Accepted: September 17, 2021; Published: September 30, 2021

\begin{abstract}
Gastric cancer (GC) is not only one of the common gastrointestinal malignancies in clinic, but also the most common cancer in the world. In recent years, its morbidity has increased. The global morbidity and mortality of gastric cancer rank fifth among all malignancies. The morbidity and mortality of gastric cancer in our country ranks second among malignant tumors, which is twice as high as the world average. In rural, the number is 1.6 times of in urban. The early diagnosis of most gastric cancers is rare in majority of patients, and most of them are in intermediate or advanced stage when they are in treatment. The proportion of radical surgery is not high, and it is easy to relapse and metastasize. The 5 -year survival rate is about $40 \%$, and the treatment effect is poor, which is severely harmful to people's health. Although the pathogenesis of gastric cancer is related to many factors, the nosogenesis is vague. The occurrence of tumors is closely related to the formation of new blood vessels. New blood vessels are the internal conditions for tumor cell proliferation and metastasis. The factors that promoting angiogenesis include more than 10 kinds of fibroblast growth factors (FGFs) and vascular endothelial growth factors (VEGF), among which VEGF is the most important regulator that promotes angiogenesis. By promoting the mitosis of vascular endothelial cells, it forms an essential matrix for the growth of vascular endothelial cells, plays a crucial regulatory role in the process of tumor angiogenesis, and participates in the occurrence and development of a variety of tumor cells. This article summarizes the research progress on the vascular endothelial growth factor's expression in gastric cancer and prognostics follows.
\end{abstract}

Keywords: Gastric Cancer, Tissue, Serum, Vascular Endothelial Growth Factor, Expression, Prognosis

\section{Introduction}

Gastric cancer (GC) is the most common cancer in the world and one of the most common gastrointestinal malignancies in clinic [1]. On the basis of the newest research production of the International Agency for Research on Cancer (IARC), there are approximately 18.1 million new cancers and 9.6 million patients who die from cancer, among which 1.03 million are gastric Cancer, accounting for $5.6 \%$ of the total number of cancers. The incidence is about $11.1 / 100,000$, ranking the fifth among all malignant tumors. The number of dying from gastric cancer is 782,000 , accounting for $8.2 \%$ of all tumor deaths, and the mortality rate is about 8.2/100,000 [2]. Cancer data in China in 2015 showed that the number of new cases and deaths are about 403,000 and 291,000 , and the morbidity and mortality are $29.31 / 100,000$ and $21.16 / 100,000$, respectively, ranking the second in malignant tumors [3],which are both twice higher than the world average level, and there are significant differences between urban and rural areas. In rural, themorbidity rate is 1.6 times of in urban [4]. Because of the stomach has a hidden disease, fetal hydrops, such characteristics as high recurrence and metastasis, most patients with gastric cancer have already in the advanced stage when they see a doctor, whose radical resection ratio is not high, and a high incidence of postoperative recurrence and metastasis, the median surial time only for $3 \sim 24$ months, 5-year survival rate was about $40 \%$ [4], treatment effect is poorer. It has become one of the malignant tumors that are severely harmful to human health. Up to now, the mechanism of occurrence and 
development of gastric cancer is still unclear. Research has proved that the occurrence, development and metastasis of tumors largely rely on the formation of new blood vessels. Various tumor cells can synthesize and secrete a variety of cytokines. Vascular endothelial growth factor (VEGF) is an important factor that promotes endothelial cell division and angiogenesis, and its expression level has closely connection to the degree of tumor vascularization [5]. VEGF is considered to be the strongest angiogenic factor and is bound up with the occurrence and development of tumors [6,7]. This article reviews the vascular endothelial growth factor's expression in gastric cancer and progress in prognostic research.

\section{The Composition and Biological Characteristics of VEGF and Its Receptors}

VEGF is a homodimeric glycoprotein composed of two identical polypeptide chains cross-linked by disulfide bonds, and its relative molecular mass is $34-45 \mathrm{KD}$. There are 6 family members of human VEGF, namely VEGF (VEGF-A), VEGF-B, VEGF-C, VEGF-D, VEGF-E and placental growth factor (PIGF), of which VEGF-A and VEGF-B are a soluble secreted protein that is a common form of VEGF [5]. VEGF mostly exists in free form, and the bound type is a reserve form. When the body's demand increases, it can be released through the regulation of proteolytic enzymes, which suggests that the biological activity of VEGF is regulated by both gene structure and proteolytic enzymes. VEGF is a prerequisite for angiogenesis. It acts specifically on endothelial cells through tyrosine kinase receptors, promotes proliferation and division, stimulates the new blood vessel formation, promotes increased vascular permeability, keeps in good condition and integrity of blood vessels, facilitates the extravasation of plasma fibrin, and promotes the formation of blood vessels. VEGF can play a part in two ways: autocrine or paracrine. Vascular endothelial growth factor receptor (VEGFR) is divided into 5 types according to its structure and function, namely VEGFR-1 (Flt-1), VEGFR-2 (Flk-1 or KDR) and VEGFR-3 (Flt-4). The first two are the most studied and the most important receptors, which are highly expressed in the vascular endothelial cells of many tumors. KDR is mainly distributed in vascular endothelial cells. It combines with VEGF-A, VEGF-C and VEGF-D to induce cell mitosis, proliferation and migration, and has an important effect on angiogenesis and growth. Under normal circumstances, KDR is expressed at a low level, which can maintain blood vessel density and permeability and is conducive to the transportation of nutrients. Significantly high expression can occur in stress or pathological conditions such as malignant tumors [8]. VEGFR-3 is mainly distributed in lymphatic endothelial cells, and plays a role through specific binding with VEGF-C and VEGF-D. The positive expression of tumor cells has a connection to lymph node metastasis.

Various types of VEGF have different not only in binding properties and affinity to their receptors, but also in the biological effects that they produce. VEGF receptors (VEGFR) exist on cell membranes, and they belong to tyrosine protein kinase receptors. When VEGFR binds to VEGF, it dimerizes and is phosphorylated to activate. The activated VEGFR transmits the signal to the cell to cause a series of biological reactions, including gene transcription. VEGF-A binds to VEGFR-1 (Flt-1) and VEGFR-2 (KDR/Flt-1), the latter being a participant in almost all cellular responses caused by VEGF [9]. VEGF-C is called lymph angiogenesis factor and is a newly discovered member of the VEGF family. VEGF-C and VEGF-D bind to VEGFR-3 to play different roles. It can bind to specific receptors of VEGFR-2 or VEGFR-3 to promote the production of blood vessels and lymphatic vessels, respectively; VEGF-C can act on VEGFR-3 through paracrine to promote its own secretion and mediate the proliferation of lymphatic endothelial cells and lymphatic hyperplasia, and regulate the differentiation of lymphatic endothelial cells. Therefore, VEGF-C is often regarded as an important generation factor of lymphatic vessel [10]. Tumor angiogenesis and lymph angiogenesis are the basic processes of blood vessel and lymph angiogenesis, whose effect allows tumors to grow and spread. VEGF and VEGF-C bind to their receptors (VEGFR-2, VEGFR-3) in endothelial cells to trigger signals that regulate this process. VEGF mainly has the following functions [11]: (1) VEGF can promote the division and proliferation of vascular endothelial cells. (2) VEGF can significantly improve the permeability of blood vessels, and its effect is far stronger than that of vasoactive substances such as histamine. (3) VEGF can promote the development of hematopoietic cells. (4) VEGF can promote the infiltration and migration of endothelial cells. (5) VEGF can accelerate the growth of lymphatic endothelial cells and induce the formation of lymphatic vessels. (6) VEGF can change the extrinsic matrix and promote the formation of vascular supports. (7) VEGF can inhibit cell apoptosis and so on. (8) VEGF induces the activation of STAT3 and then activates the STAT3 signaling pathway [12], promoting the self-renewal of cancer stem cells [13].

The expression of VEGF's receptors is adjusted to many factors. Hypoxia is the most important factor, followed by oncogenes, tumor suppressor genes and cytokines. Hypoxia can increase the stability of VEGF mRNA, increase the expression of VEGFR, and enhance the biological effects of VEGF. Once hypoxia is improved, the mRNA level of VEGF is reversibly down-regulated. The influence mechanism of VEGF on tumors may have the following aspects [5] :(1) Promoting tumor angiogenesis is related to VEGF increasing vascular permeability, promoting the proliferation of vascular endothelial cells and the growth of vascular supports. (2) The promotion of tumor lymphatic growth is related to the joint involvement of VEGF-C, VEGF-D and VEGFR-3 in tumor growth and lymphatic metastasis. (3) Influencing tumor cell dynamics is related to VEGF inducing the expression of anti-apoptotic protein Bcl-2 and inhibiting tumor cell apoptosis. (4) Enhancing the tolerance of tumor cells to radiotherapy is related to the effect of VEGF on the formation of blood vessels and inducing the differentiation and 
maturation of hematopoietic stem cells. Research has shown that the formation of tumor blood vessels is adjusted to many factors. Among them, VEGF is the most important factor that has been found to promote tumor blood vessel formation. Overexpression of VEGF and its receptor is found in many human solid tumors.

\section{The Expression of VEGF in Gastric Cancer Tissue}

The positive expression rate of VEGF in GC varies greatly. Maeda et al. report that the positive rate of VEGF in GC tissue is $43.4 \%(56 / 129)$, which is mainly located on the cytoplasm and membrane of cancer cells. Strong positive expression is more common in the front edge of tumor infiltration, and some endothelial cells show weak positive expression, and there is no VEGF expression in normal gastric mucosa. Yamamoto et al. use in situ hybridization to detect the expression of VEGF mRNA in 8 gastric cancer cell lines, $30 \mathrm{GC}$ tissues and normal gastric mucosa, finding that all cancer cell lines express VEGF mRNA, and its level is closely related to the content of VEGF secreted into the culture medium; The expression of VEGF mRNA in GC tissue is higher than normal gastric mucosa, and the expression of advanced well-differentiated gastric adenocarcinoma is higher than early well-differentiated gastric adenocarcinoma. Lieto et al. [14] find that the expression rate of VEGF in GC tissue is $47.7 \%(42 / 88)$. Using qRT-PCR and immunohistochemical methods to detect the mRNA and protein expression levels of CRMP4, VEGF, and VEGFR2, finding that the expression of VEGF and CRMP4 in GC tissues is apparently higher than adjacent tissues [15]. Zhao et al. [16] report that the positive expression rate of VEGF in GC tissue $(70.1 \%, 75 / 107)$ is obviously higher than gastric mucosa $(20.0 \%, 6 / 30)$, and the expression of SSTR in GC tissues $(62.6 \%)$ is apparently lower than normal tissues (93.3\%). Zhou Qiang [17] report the positive expression rate of VEGF in GC tissue is $62.4 \%$ (78/125), which is obviously higher than in adjacent tissues of $24.0 \%$. Liao Jie et al. [18] report that the VEGF's positive rate in GC tissue $(48.75 \%$, $39 / 80)$ is significantly higher than the control group $(10.00 \%$, $2 / 20),(\mathrm{P}<0.05)$. The positive expression rates of VEGF and p53 in GC tissue are $61.25 \%$ and $58.75 \%$, respectively, as are apparently higher than the $40.00 \%$ and $37.50 \%$ of chronic atrophic gastritis (CAG). The difference has statistically significant $(\mathrm{P}<0.05)$, indicating that the VEGF's high expression and p53's high expression is correlated with the occurrence of CAG and GC. The expression of p53 and VEGF in the CAG group are lower than the GC group and higher than the control group, and the differences have statistical significance $(\mathrm{P}<0.05)$ [19]. Combined immunohistochemistry and ELISA are used to detect the VEGF-C's expression in 98 cases of GC tissues. The positive expression rate $(74.5 \%$, $73 / 98$ ) is apparently higher than normal gastric mucosal tissues $(61.1 \%, 22 / 36)$. The difference has statistical significance $(\mathrm{P}<0.01)$ [20]. The VEGF-C's positive expression rate in advanced GC tissue $(81.25 \%)$ is obviously higher than the gastritis group $(7.50 \%, \quad \mathrm{P}<0.01)$ [21]. Meta-analysis results show that the VEGF-C protein's positive expression rate in GC tissue is $59.51 \%$ (463/778). It is significantly higher than normal gastric tissue $(8.62 \%$ $(27 / 313)$, and the difference has statistical significance $(\mathrm{P}<0.05)$ [22]. Zhang Yinghui et al. [23] report that the positive expression rates of cyclooxygen-ase-2 (COX-2) protein and VEGF-C protein in the GC group are $63.08 \%$ and $61.29 \%$, respectively, which are obviously higher than the precancerous lesion group (36.00\% and $28.00 \%)$ and the control group $(\mathrm{P}<0.05)$. The COX-2 protein's expression in GC tissue is positively connected to the expression of VEGF-C protein $(\mathrm{r}=0.411, \mathrm{P}<0.05)$. Liu Jie [24] et al. transfect human gastric cancer cell BGC-823 with HMGB1 recombinant plasmid, and analyze the expression of VEGF-D mRNA and the correlation by reverse transcription PCR. It is found that the VEGF-D's positive rate in GC tissue $(55.2 \%$, $48 / 87)$ is apparently higher than in adjacent tissues $(25.3 \%$, $22 / 87$ ), while no staining positive cells are found in normal gastric mucosa. VEGF-D in GC tissue D is apparently higher than adjacent tissues and normal gastric mucosal tissues (all $\mathrm{P}<0.05$ ). The results of Spearman rank correlation analysis show that the HMGB1's positive expression in GC tissues is positively connected to VEGF-D ( $\mathrm{r}=0.482, \mathrm{P}=0.001)$, which are obviously higher than in adjacent tissues. In vitro research has confirmed that HMGB1 can promote the transcription of VEGF-D gene, and may play a synergistic role in the pathogenesis of GC. The above research results show that the VEGF, VEGF-C and VEGF-D protein's expression in GC tissues are higher than those in the control group, which suggests that they have a great importance in the development of GC and participate in the development of GC.

\section{VEGF and the Prognosis of Gastric Cancer}

There is much research on the connection of VEGF expression and the invasiveness of gastric cancer, but results are not the same. Maeda et al. find that VEGF expression has nothing to do with the depth of invasion, tissue stage and differentiation type of gastric cancer, but is significantly related to lymphatic and blood vessel invasion and lymph node metastasis. Tanigawa et al. find that VEGF expression is associated with the classification and tissue type of gastric cancer, but there is no connection among the depth of invasion, lymph node metastasis, lymphatic and vascular infiltration. However, Saito et al. believe that the VEGF's expression is apparently connection to the depth of invasion of gastric cancer. Tao Houquan et al. [25] use immunohistochemical techniques to detect the VEGF's expression in 128 cases of GC specimens, finding that the MVC of the positive VEGF expression is apparently higher than the negative. The degree of VEGF expression is significantly related to tumor invasive growth, serosal infiltration, lymph node metastasis and liver metastasis. The recurrence rate of VEGF-positive patients is higher than that of negative patients, and the prognosis is poor. 
Yang et al. [26] find that the MMP-9 mRNA's expression is positively connected to VEGF protein and microvessel density (both $\mathrm{P}<0.001$ ), and the positive rate of VEGF in GC patients with vascular infiltration, lymph node metastasis, liver and peritoneal metastasis in T3 T4 stage is significantly higher than those in T1 T2 stages and without vascular infiltration, lymph node metastasis, liver and peritoneal metastasis. Tumor angiogenesis is more active in patients with high VEGF expression, and the 5-year survival rate is lower. The expression of MMP-9 and VEGF is related to the enhancement of tumor angiogenesis, and may play an important role in the invasion and metastasis of GC. Therefore, MMP-9 and VEGF may be biological indicators for judging the prognosis of GC patients, and also be a new target for GC treatment. The VEGF and VEGFR's positive expression in gastric adenocarcinoma tissue can judge its pathological stage, metastasis and prognosis. After tumorigenesis, the VEGF's expression significant increase, and the expression of VEGF is closely connected to tumor stage, distant metastasis, lymph node metastasis, tumor diameter, etc., but has no obvious relationship with tumor location, degree of differentiation, and tissue type [27]. Chen [28] et al. find that the galectin-1 and VEGF's expression in GC patients is positive connection, and the positive rates are $63.0 \%(68 / 108)$ and $57.4 \%(62 / 108)$, respectively. The expression of the former is connected with tumor size, degree of differentiation, TNM stage, lymph node metastasis and VEGF expression, while the expression of the latter is related to tumor size, TNM stage, and lymph node metastasis. Kaplan-Meier survival analysis shows that both high expressions are significantly correlated with poor prognosis of GC. Multivariate analysis shows that the expressions of both are independent prognostic parameters of overall survival in GC patients, and could be used as independent prognostic indicators of poor GC survival. It shows that the high expression of galectin-1 and VEGF has more to do with the biological behavior of GC. The HPIAS-1000 high-definition color pathology graphic report analysis system is used to quantitatively analyze the immunohistochemical staining results, and it is found that the VEGF level of GC tissue $(135.84 \pm 12.67)$ is apparently higher than of healthy people $(86.56 \pm 10.36, \mathrm{P}<0.05), \mathrm{COX}-2$. The expression of VEGF and MMP-9 also change significantly. The expression of COX-2 may promote the VEGF and MMP-9's expression, and accelerate the formation, invasion and metastasis of tumor blood vessels in patients [29]. The expression of CD11c and VEGF in GC and adjacent tissues and serum VEGF concentration are detected by immunofluorescence and ELISA. It is found that the intensity of VEGF expression in GC tissue is negatively connected with the density of dendritic cells (DCs) in the tumor. The number of VEGF-positive DCs is positively correlated with the progression of GC, while the serum VEGF concentration has nothing to do with the density of DCs infiltration in tumors [30], which suggests that the secretion of VEGF in GC tissues can inhibit the activity of DCs, thereby mediating the immune escape and infiltration of tumor cells. DCs in GC tissues can also secrete VEGF to participate in disease progression. Pang et al. [31] find that VEGF expression in GC stage-N3 patients is approximate 7 times higher than N0 patients, and increased with the increase of lymph node metastasis (P\&LT; 0.01). VEGF expression in tumor cells with distant metastasis is apparently higher than tumor cells without distant metastasis (P\&LT; 0.01). The VEGF's expression in stage-T4 stage of $\mathrm{GC}$ is about 10 times that of stage-Tis stage GC, and the deeper the tumor cell infiltration, the higher the expression level of VEGF $(p<0.01)$. The VEGF's expression in GC tissue is positively connected with the depth of tumor cell invasion, TNM stage, lymph node metastasis and distant metastasis. With the disappearance of tumor symptoms, the patient's VEGF decreases, indicating that VEGF is likely to become the main factor predicting the recurrence of GC. Jayson [32] and Wang [6] find that the VEGF's expression in GC tissue is connected to TNM staging, lymph node metastasis, and degree of venous invasion. Most patients with positive expression of VEGF have a poor prognosis. Chen Xiujiao et al. [33] find that the positive expression rate of VEGF in GC tissue $(68.0 \%)$ is obviously higher than the positive expression rate in adjacent tissues $(10.0 \%)$, and between both has the significance difference $(\chi 2=33.164, \mathrm{P}<0.05)$. TNM staging is closely connected with lymph node metastasis $(\mathrm{P}<0.05)$. CD147 is an extracellular matrix metalloproteinase inducing factor and also a cell surface adhesion molecule. The expression of CD147 and VEGF in GC tissue is positively correlated, and is related to TNM staging and lymph node metastasis $(\mathrm{P}<0.05)$, but there is no connection among gender, age, and degree of differentiation. The detection of CD147 and VEGF expression is of great significance for judging the malignant degree of gastric cancer, predicting its invasion, metastasis and prognosis, and guiding treatment. The immunohistochemical SP method is further used to detect the Girdin and VEGF's expression in 100 cases of GC tissues and their distribution in different pathological characteristics. It is found that the positive expression rates of Girdin and VEGF in GC tissues are $47.0 \%$ and $65.0 \%$, respectively, and both are related to lymph nodes. No metastasis is related to different TNM pathological stages, but there is statistically insignificant difference with age, gender and degree of differentiation $(\mathrm{P}>0.05)$. The positive expression rate of VEGF in GC tissues with lymph node metastasis $(82.2 \%)$ is higher than those without metastasis $(18.5 \%, \mathrm{P}<0.05)$, and the positive expression rate of VEGF $(81.6 \%)$ in stage III-IV is higher than that in stage I-II $(49.0 \%, \mathrm{P}<0.05)$. Spearman correlation analysis find that the expression of Girdin and VEGF in GC tissue is positively correlated $(\mathrm{P}<0.05)$ [34]. The combined detection of Girdin and VEGF expression in GC tissue has certain value for judging the degree of malignancy, predicting invasion and metastasis and prognosis, and guiding treatment. Liao Jie et al. [18] report that the VEGF's positive expression rate in $\mathrm{GC}$ tissue is apparently higher than that in the control group $(\mathrm{P}<0.05)$, and it is not related to gender, age, maximum tumor diameter, tumor location, and vascular tumor thrombus $(\mathrm{P}>0.05)$. It has something to do with the degree of differentiation of GC tissue, depth of invasion, lymph node metastasis, and TNM staging $(\mathrm{P}<0.05)$. The MST of GC 
patients with VEGF-positive and VEGF-negative patients is 18.27 and 58.00 months, respectively, and between both has the significance difference $(\mathrm{P}=0.000)$. Univariate analysis shows that VEGF is closely connected to the prognosis of GC patients $(\mathrm{P}<0.05)$. Cox regression model analysis shows that the degree of differentiation, TNM stage, VEGF, and vascular tumor thrombus are independent prognostic factors for GC $(\mathrm{P}<0.05)$. Cox multivariate analysis shows that VEGF is an independent prognostic factor of $\mathrm{GC}(\mathrm{P}<0.05)$.

Immunohistochemical method is applied to analyze the relationship between the expression levels of VEGF-A and cell membrane skeletal protein $\alpha$-Adducin (ADD1) and the pathological characteristics and prognosis of GC. It is found that the positive expression rate and level of VEGF-A in GC tissues are apparently higher than in adjacent tissues. Statistical significance exists in the difference $(P<0.05)$. The VEGF-A's expression is correlated with the location and prognosis of $\mathrm{GC}$, and the ADD1's expression is connected with the degree of differentiation of GC tissue, TNM stage, depth of invasion, lymph node metastasis and prognosis. VEGF-A and ADD1 are highly expressed in GC tissues, and the two are positively correlated. OS and DFS in the high expression group of VEGF-A and ADD1 are obviously lower than the low expression group of VEGF-A and ADD1 $(\mathrm{P}<0.05)$ [35]. The research finds that the VEGF-C's expression rate in the COX-2 positive group in GC tissue is $74.46 \%(382 / 513)$, which is significantly higher than $64.91 \%(172 / 265, \mathrm{P}<0.05)$ in the COX-2 negative group [22]. It is suggested that COX-2 and VEGF-C are over-expressed in GC tissues, which are closely related to GC lymphatic metastasis, and may play a part in promoting the formation of GC microlymphatic vessels. The positive expression rates of COX-2 and VEGF-C protein in GC patients are apparent higher than in the precancerous lesion group and the control group $(\mathrm{P}<0.05)$. The positive expression rate of COX-2 protein in patients with TNM stages I II, well-differentiated and non-lymph node metastasis patients are obviously lower than in TNM stages III IV, moderately and poorly differentiated patients with lymph node metastasis $(\mathrm{P}<0.05)$. The positive expression rate of VEGF-C protein in patients with TNM stage I II and without lymph node metastasis is apparently lower than patients who are in TNM stage III IV and have lymph node metastasis $(\mathrm{P}<0.05)$. The median survival time (MST) of GC patients in the COX-2(+)VEGF-C $(+)$ group and COX-2(+) group are 22 months and 21 months, respectively, which is apparently lower than that of $\mathrm{VEGF}-\mathrm{C}(+)$ Group and COX-2(-)VEGF-C(-) group $(\mathrm{P}<0.05)$. It is suggested that COX-2 and VEGF-C play an important part in GC and precancerous lesions. COX-2 and VEGF-C are associated with GC lymph node metastasis, clinical stage and degree of differentiation and COX-2 is also linked to the prognosis of patients [23]. Immunohistochemical SP method and ELISA are used to detect the levels of IL-33 and VEGF-C in gastric mucosal tissue specimens and serum of $98 \mathrm{GC}$ patients and 36 healthy subjects. It is found that the expressions of both in GC tissues are apparently higher than normal gastric mucosa Tissue. The difference among different tumor differentiation degree, tissue invasion, lymph node metastasis, distant metastasis and clinical stage has statistically significant $(\mathrm{P}<0.05)$, and the positive expression of lymph node metastasis is higher than non-metastasis $(\mathrm{P}<0.05)$ [20]. Immunohistochemical method is used to detect the expression of stem cell transcriptional regulator Sox2 (sex determining region Y-box2) and VEGF-C protein [36], and it is found that the positive expression rate of Sox 2 protein in GC patients $(57.69 \%)$ is apparently lower than normal gastric mucosa group and chronic gastric mucosa group. In the atrophic gastritis group $(\mathrm{P}<0.05)$, the VEGF-C protein's positive expression rate $(75.00 \%)$ is obviously higher than the normal gastric mucosa group and chronic atrophic gastritis group $(\mathrm{P}<0.05)$. There is no significant difference in the positive expression rate of Sox 2 and VEGF-C between the GC group and the atrophic gastritis with intestinal metaplasia or dysplasia group $(\mathrm{P}>0.05)$. TheSox 2 protein's positive expression rate in patients with mucinous adenocarcinoma, TNM stages III to IV, moderately poorly differentiated, and lymph node metastasis is significantly lower than non-mucinous adenocarcinoma, TNM stages I to II, well differentiated and non-lymph node metastasis patients $(\mathrm{P}<0.05)$. The positive expression rate of VEGF-C protein in patients with serous membrane invasion and lymph node metastasis is apparently higher than patients without serous membrane invasion and no lymph node metastasis $(\mathrm{P}<0.05)$. There is no correlation between Sox 2 and VEGF-C protein expression in GC tissue $(\mathrm{P}>0.05)$. It is suggested that Sox2 protein is low in GC tissue, while VEGF-C protein is highly expressed, which is associated with the clinicopathological characteristics of patients and may be involved in the development of GC. The immunohistochemical SP method is used to detect the expression of COX-2, VEGF, and VEGF-C protein in gastric cancer, and it is found that the positive expression rate of the three in GC tissue is higher than in adjacent tissues $(\mathrm{P}<0.05)$. The positive expression rate of VEGF and VEGF-C in well-medium differentiated tissues is lower than in poorly differentiated GC tissues $(\mathrm{P}<0.05)$. TNM stage is divided into III and IV. The positive expression rate of COX-2, VEGF, VEGF-C in GC tissue with lymphatic metastasis is higher than in GC tissue with grade I and II and without lymphatic metastasis $(\mathrm{P}<0.05)$ [37].It is suggested that the over-expression of COX-2, VEGF, and VEGF-C play an important part in the formation of tumors and can promote the development of GC. The combined detection of the three can be used as a reference for the pathology of gastric cancer and the prognosis of patients. The VEGF-D's expression in gastric cancer tissue is obviously higher than in the control group, and is positively correlated with MMP-11 and MMP-12.The prognosis of GC patients with high expression of the three is poor [38], and the combined detection may have a certain value in judging the prognosis. The above research results indicate that VEGFs is involved in the development of $\mathrm{GC}$, and participate in and play an important role in the formation, infiltration, development and metastasis of GC tumors together with MMP-9, COX-2, Girdin, ADD1, Sox2, etc, but it is different from the biological behavior of GC. 


\section{The Relationship Between Serum VEGF Level and Prognosis of Gastric Cancer}

Most malignant tumors are often accompanied by increased secretion of VEGF, which suggests that elevated serum VEGF levels have a poor prognosis. Serum VEGF level can be used as a tumor marker (Tumor marker, TM), which has important value for tumor diagnosis and prognosis judgment. The level of serum VEGF in GC patients significantly increases, and is related to the TNM staging of GC. The advanced stage is significantly higher than that of the early stage. The serous membrane infiltrates are significantly higher than the non-serosa infiltrated patients. There is also statistical significance in the depth of venous invasion and tumor invasion [39]. The serum VEGF level is also related to the recurrence of $\mathrm{GC}$ and the effect of chemotherapy. The serum VEGF level of relapsed patients is higher than that of advanced stage, and those who are effective in chemotherapy will decrease, and those who are not effective will increase. But the serum VEGF level has nothing to do with histological type and degree of differentiation. Zhou Haiyin et al. [40] find that GC cell differentiation being low, lymph node metastasis, infiltration and penetration of serosal membrane, tumor $\geq 5 \mathrm{~cm}$ and serum VEGF levels in patients with stage III and IV significantly increase, which suggests that changes in serum VEGF levels can reflect GC degree of progress. The high level of serum VEGF before surgery and the VEGF's positive expression in tumor tissues are closely related to tumor metastasis, staging and prognosis. Therefore, the detection of serum VEGF can not only judge the stage and prognosis of the tumor, but also dynamic monitoring can help judge the curative effect, recurrence and metastasis. Hu Min et al. [41] report that the serum VEGF level of GC patients is apparently higher than normal controls, and the postoperative VEGF level is significantly lower than that of preoperative. Its level is closely related to the size of the GC tumor, the depth of invasion, and the degree of lymph node involvement. With the increase of clinical pathological stage, the level of VEGF also increases significantly, but it has nothing to do with the degree of histological differentiation and gender. Therefore, the detection of preoperative serum VEGF levels is of great significance in predicting the invasion and metastasis of GC. Kang Shirong et al. [42] report that the preoperative serum VEGF level of GC patients is higher than that of the control group, which is positively correlated with gastric cancer tissue microvessel density (MVD) ( $\mathrm{r}=0.875$ ), serum VEGF level, MVD and GC infiltration depth in cancer tissues, and lymph nodes. There are significant differences in metastasis and histological classification, regardless of gender and age. It is suggested that the angiogenesis of GC is closely related to the level of serum VEGF, and the level of serum VEGF can be used as an important indicator to evaluate the efficacy, recurrence, metastasis and prognosis of GC.

Mysliwiec et al. [43] find that the serum VEGF-A level of patients with intestinal and diffuse GC increases, but the level of VEGFR-2 does not change significantly. The serum VEGFR-2 level of patients without metastasis after surgery is higher than that before surgery, and those with lymphoma GC patients have higher serum VEGF and VEGFR-2 levels. Hălmaciu et al. [44] find that the preoperative serum VEGF-A level $(376 \mathrm{pg} / \mathrm{ml})$ of GC patients is apparently higher than normal controls $(157.3 \mathrm{pg} / \mathrm{ml})$, and its level is associated with the type of tumor tissue, and the serum VEGF-A level is higher than the survival rate of patients with $173 \mathrm{pg} / \mathrm{ml}$ is higher, so VEGF-A cannot be used as a predictor of GC, but it can be used as a marker of disease progression. Liu et al. [45] conduct a meta-analysis of 44 published studies (including 4794 patients with gastric cancer undergoing surgical resection) and find that no matter what the high expression of VEGF in GC tissues or the increase of serum VEGF, VEGF-C, VEGF-D levels, they are all related to the poor prognosis after GC. Serum VEGF may be better than tissue VEGF in predicting prognosis. Therefore, the increase of serum VEGF level is an independent indicator of patient survival and judging tumor type and depth of invasion. Villarejo-Campos et al. [46] compare the preoperative and postoperative serum VEGF and VEGF-C levels of $59 \mathrm{GC}$ patients to explore their relationship with prognosis and survival. The monthly serum VEGF and VEGF-C are measured, and it is found that the serum VEGF level decreases after tumor resection. The survival rate of patients with preoperative VEGF level $>761 \mathrm{pg} / \mathrm{ml}$ is lower than that of those with less than this value. Multivariate statistical analysis finds that serum VEGF level is an independent prognostic factor for gastric cancer. Serum VEGF level is related to tumor type and surrounding tissue infiltration. Serum VEGF is positively correlated with carcinoembryonic antigen levels [47].Serum VEGF, MMP-9, and endothelin levels in GC patients are obvious superior to the control group, and are related to the clinical stage of GC, lymph node metastasis, tumor diameter, and depth of invasion, which indicates that the above three are involved in the development of GC. Cell growth, invasion and metastasis play an important role and are closely associated with tumor progression [48]. Serum VEGF levels in GC patients can provide additional prognostic information for preoperative assessment of invasiveness and tumor type. Serum IL-33 and VEGF-C levels in GC patients are $50.24 \pm 13.08 \mathrm{pg} / \mathrm{mL}$ and $210.73 \pm 58.35 \mathrm{pg} / \mathrm{mL}$, respectively, which are superior to those who have healthy physical examination $(\mathrm{P}<0.05)$, and those with lymph node metastasis are higher than those without lymph node metastasis $(\mathrm{P}<0.05)$ [20]. It is suggested that the high level of IL-33 in the serum of GC patients may induce the secretion of VEGF-C and promote GC lymph node metastasis, which can be used as a major indicator for evaluating the prognosis of GC. Yao Jingjing [49] explore the clinical significance of the combined detection of serum IL-6, carbohydrate antigen 724 (CA724), and VEGF in GC patients, and find that the serum levels of IL-6, CA724 and VEGF in the liver metastasis-positive group are obviously higher than those in the liver metastasis-negative group. And 
the liver metastasis negative group is apparently higher than the control group. The sensitivity, specificity and diagnostic coincidence rate of the combined detection of serum IL-6, CA724 and VEGF in GC patients are apparently higher than the single detection $(\mathrm{P}<0.05)$. It is suggested that the combined detection of serum IL-6, CA724, and VEGF can effectively improve the diagnostic sensitivity, specificity and coincidence rate, and has high application value for the diagnosis of GC. Liu Bing et al. [50] find that the levels of bFGF, VEGF, and CE are closely associated with the prognosis of $\mathrm{GC}$, and their low levels indicate that the patient has a positive effect and a better prognosis. The effect of advanced GC is higher than the control group $(\mathrm{P}<0.05)$. The postoperative follow-up showed that the 3 -year and 5 -year survival rates of the patients are (54\% and $40 \%),(36 \%$ and $17 \%)$, respectively, and there is a significant difference $(\mathrm{P}<0.05)$. Dong Lei et al. [51] find that the preoperative serum VEGF, MMP-2, and MMP-9 levels of 84 GC patients are apparently higher than the control group, and are apparently lower than the preoperative 7 days after surgery $(\mathrm{P}<0.05)$. The GC lymph node metastasis group is significantly higher than the non-lymph node metastasis group $(\mathrm{P}<0.05)$. The combined detection of three indicators for ROC curve analysis shows that the sensitivity of diagnosing GC lymph node metastasis is $78.95 \%$, $63.16 \%$, $73.68 \%$, and $97.37 \%$, respectively, and the specificities are $95.65 \%, 91.30 \%, 93.48 \%$ and $97.83 \%$, respectively. It is suggested that preoperative serum VEGF, MMP-2, and MMP-9 levels can be used as TM for the diagnosis of GC lymph node metastasis. Combined detection for GC lymph node metastasis has the highest diagnostic value. Dynamic detection is beneficial to judge the prognosis and provides a basis for postoperative treatment strategies.

\section{Conclusion}

GC is one of the most common malignancies. The TM currently used for GC examination mainly includes CEA, CA19-9, CA72-4 and CA242.Because of TM detection being simple, non-invasive, reproducible, and easy to accept by patients, GC markers are widely used in clinical prognosis judgments of GC. Research have confirmed that VEGF is the most important regulator of angiogenesis, which promotes the formation of tumor blood vessels by stimulating the proliferation of vascular endothelial cells and the increase of vascular permeability, and features in the occurrence, development and metastasis of GC. The expression of VEGF and its receptors in $\mathrm{GC}$ tissues and changes in serum levels are closely associated with their biological behaviors. A number of researches have shown that VEGF is expressed at a high level in GC tissue and serum, and its expression is positively connected with GC pathology and clinical staging. Detecting the expression of VEGF and its receptor in GC tissue and serum VEGF level is of great value for the diagnosis and prognostic monitoring of GC. It provides an important basis for judging the recurrence after GC and formulating individualized treatment plans, but the results of the research are not the same. TM detection features in the prognostic judgment of GC. In recent years, there have been many researches on combined detection of serum VEGF and other $\mathrm{TM}$ for GC diagnosis and prognosis judgment, which has improved the sensitivity and specificity of diagnosis and prognosis judgment, and opened up a new way for GC research. Based on the principles of evidence-based laboratory medicine, it is imperative to screen several tumor markers such as serum VEGF with high sensitivity and specificity for GC diagnosis and prognosis.

\section{References}

[1] Hou JZ, Li Y. Relationship Between the Expression of S100P in Gastric Cancer Tissue and Clinical Pathology [J]. American Journal of Clinical and Experimental Medicine, 2021, 9 (3): 55-64.

[2] Bray F, Ferlay J, Soerjomataram I, et al. Global cancer statistics 2018: GLOBOCAN estimates of incidence and mortality worldwide for 36 cancers in 185 countries [J]. CA Cancer J Clin. 2018, 68 (6): 394-424.

[3] Zhou JS, Zheng RS, Zhuang GH, et al. Analysis on the trend of gastric cancer incidence and age change in cancer registration regions of China [J]. J Applied Oncology 2000 to 2015 [J]. 2020, 34 (1): 1-5.

[4] Song ZH, Xu XN, Li KL, et al., Research progress on etiology of gastric cancer [J]. Chin Med J Metall Indus, 2020, 37 (5): 509-511.

[5] Yang L, Hou JZ, Hou ZJ. Research progress of vascular endothelial growth factor and its receptor in gastric cancer [J] Chin Med Equip. 2014, 11 (4): 68-69.

[6] Wang C, Li J, Ye S, et al. Oestrogen inhibits VEGF expression and angiogenesis in triple-negative breast cancer by activating GPER-1 [J]. J Cancer, 2018, 9 (20): 3802-3811.

[7] Wu JB, Tang YL, Liang XH. Targeting VEGF pathway to normalize the vasculature: an emerging insight in cancer therapy [J]. Onco Targets Ther, 2018, 11: 6901-6909.

[8] Yang YQ, Song ZY. The role of vascular endothelial growth factor receptor (KDR) in infiltration and metastasis of gastric cancer [J]. 2011, 49 (27): 98-99, 101.

[9] LuoYF, Huang HY. Relationship between serum soluble vascular endothelial growth factor and its receptor and tumor [J]. Chin Clin New Med, 2014, 7 (8): 779-783

[10] Xu WZ, Liu M, Wang ZR. Effect of Xiaoyutang on serum VEGF protein content in patients with advanced gastric cancer after operation [J], Trad Chin Med, 2016, 39 (2): 425-427.

[11] MaL. Biological characteristics of VEGF and its receptor and its role in tumor angiogenesis [J]. J Chin Euge\& Gene, 2014, 26 (5): 146-148.

[12] Wang T, Liu J, Xiao XQ. Cantharidin inhibits angiogenesis by suppressing VEGF-induced JAK1/STAT3, ERK and AKT signaling pathways [J]. Arch Pharm Res. 2015, 38 (2): 282-289. 
[13] Byun HJ, Darvin P, Kang DY, et al. Silibinindownregulates MMP2 expression via Jak2/STAT3 pathway and inhibits the migration and invasive potential in MDA-MB-231 cells [J]. Oncol Rep, 2017, 37 (6): 3270-3278.

[14] Lieto E, Ferraraccio F, Orditura M, et al. GaliziaG. Expression of vascular endothelial growth factor (VEGF) and epidermal growth factor receptor (EGFR) is an independent prognostic indicator of worse outcome in gastric cancer patients [J]. Ann Surg Oncol, 2008, 15 (1): 69-79.

[15] Sile Chen, Xinhua Zhang, Jianjun Peng, et al. VEGF promotes gastric cancer development by upregulating CRMP4 [J]. Oncotarget. 2016, 7 (13): 17074-17086.

[16] Zhao DQ, Chen J, Wu YF, et al. Correlation between Vascular Endothelial Growth Factor and Somatostatin Receptor with Progression and Prognosis in Gastric Cancer [J]. Hepatogastroenterology, 2014, 61 (132): 1154-1158.

[17] Zho UQ, Zhang HY. Expressions of HER-2 protein and VEGF in gastric cancer tissues and their mutual relationships $[\mathrm{J}]$. $\mathrm{J}$ North Sichuan Med College2016, 31 (5): 690-692.

[18] Liao J, Yang XP Expression of VEGF in gastric carcinoma and its relationship with clinicopathological features and prognosis [J]. Clin Med Rese and Prac, 2018, 3 (9): 25-27.

[19] Li J. Expression of VEGF and p53 in chronic atrophic gastritis and gastric cancer [J]. J Henan Med Colle, 2018, 30 (2): $113-115$.

[20] Xia BX, Li F, Xu J. et al. Expression of interleukin-33 and vascular endothelial growth factor $\mathrm{C}$ in gastric cancer and its clinical significance $[\mathrm{J}]$. Chong Qing Med, 2016, 46 (15): 2056-2059

[21] Fan ZJ, Zou YJ, Zheng WL. VEGF-C protein expression and its clinical significance in patients with advanced gastric cancer [J]. OncoProg, 2017, 15 (12): 1420-1422.

[22] Liao XW, Huang BF, Xiao LH, et al. The Meta-analysis of the Expression of COX-2, VEGF-C in Gastric Carcinoma and Their Correlations with Lymphnode Metastasis [J]. Chin Fore Med \&Rese, 2017, 15 (26): 8-10.

[23] Zhang YH, Yan, W, QiaoBJ, et al. Expression and significance of COX-2 and VEGF-C protein in gastric cancer and precancerous lesions [J]. J Clin\&Expe Med 2018, 17 (10): 1040-1043.

[24] LiuJ, JiZG, BiCY, et al. Expression and correlation of HMGB1 and VEGF-D in gastric carcinoma tissue [J]. J Clin\&Expe Med, 2017, 16 (18): 1796-1800.

[25] Tao HQ, Lin YJ, Wang RN, et al. Role of vascular endothelial growth factor expression in infiltration and metastasis of gastric cancer [J]. J Chin Dige, 1998, 18 (5): 287-292.

[26] Yang Q, Ye ZY, Zhang ZX, et al. Expression of matrix metalloproteinase-9 mRNA and vascular endothelial growth factor protein in gastric carcinoma and its relationship to its pathological features and prognosis [J]. Anat Rec (Hoboken), 2010, 293 (12): 2012-2019.

[27] Zhao Y, Wang YZ. Expression and clinical significance of E-cadherin, MMP-2 and MMP-9 in gastric cancer [J]. Jiang Su Med, 2013, 39 (9): 1034-1036, 1117.

[28] Chen J, Tang D, Wang S, et al. High expressions of galectin-1 and VEGF are associated with poor prognosis in gastric cancer patients [J]. Tumour Biol. 2014, 35 (3): 2513-2519.
[29] Yan XF. Expression and clinical significance of COX-2, VEGF and MMP-9 in gastric cancer [J]. Chin Med Guide, 2016, 14 (10): 98-99.

[30] Yang F, Li AM, Jing DH. Correlation between expression of VEGF and DCs infiltration density in gastric cancer tissues [J]. J Hainan Med Univ, 2016, 22 (1): 1-4.

[31] Pang L, Wang J, Fan Y, et al. Correlations of TNM staging and lymph node metastasis of gastric cancer with MRI features and VEGF expression [J]. Cancer Biomark. 2018, 23 (1): 53-59.

[32] Jayson GC, Zhou C, Backen A, et al. Plasma Tie2 is a tumor vascular response biomarker for VEGF inhibitors in metastatic colorectal cancer [J]. Nat Commun, 2018, 9 (1): 4672.

[33] Chen XJ, Xu L, Chen ZW, et al. Expression of CD147 and VEGF in Gastric Carcinoma and its Clinical Significance [J]. J Jinzhou Med Univ, 2018, 39 (5): 1-3.

[34] Chen XJ, Li HC, XuL, et al. Expression and clinical significance of Girdin and VEGF in gastric cancer [J]. J Qiqihar Med Univ, 2019, 40 (1): 1-3.

[35] LiL, Gu HZ. Expression of vascular endothelial growth factor-A and $\alpha$-Adducin in gastric cancer and its correlation with clinicopathological features and prognosis [J]. Int J Dig Dis, 2019, 39 (3): 216-221.

[36] Zhao ZY, Su JM, Cheng ZQ, et al. Expression of Sox2 and VEGF-C in gastric cancer and its clinical significance [J]. Expe Lab Med, 2019, 37 (4): 747-750.

[37] Pu XY, Zhang YQ, Jia HY. Expression of COX-2, VEGF and VEGF-C protein in gastric cancer and its clinical significance [J]. Chin Fore Med \& Rese, 2018, 16 (20): 53-54.

[38] $\mathrm{Hu}$ NJ, Wang HL. Expression and clinical significance of MMP-11, MMP-12 and VEGF-D in gastric adenocarcinoma [J]. J Shanxi Med, 2019, 48 (5): 554-560.

[39] Karayiannakis AJ, Bolanaki H, Syrigos KN, et al. Serum vascular endothelial growth factor levels in pancreatic cancer patients correlate with advanced and metastatic disease and poor prognosis [J]. Cancer Lett, 2003, 94 (1): 119-124.

[40] Zhou HY, Ye GX, Wang SW, et al. Clinical study of VEGF levels in serum and tumor tissue of patients with gastric cancer [J]. JWorld Onco, 2004, 39 (4): 349-350, 359.

[41] Hu M, Fan ZL, Wang XH. Expression and significance of vascular endothelial growth factor in serum of patients with gastric cancer [J]. J Qinghai Med Colle, 2008, 29 (1): 36-38.

[42] Kang SY, Li J. Study on the expression of VEGF in serum and MVD in cancer tissue of patients with gastric cancer [J]. J Inner Mongolia Med Colle, 2009, 31 (1): 13-18.

[43] Mysliwiec P, Pawlak K, Bandurski R, et al. Soluble angiogenesis markers in gastric tumor patients [J]. Folia Histochem Cytobiol, 2009, 47 (1): 81-86.

[44] Hălmaciu I, Gurzu S, Dobreanu M, et al. The most studied VEGF molecule is VEGF-A (Vascular Endothelial Growth Factor). Its involvement in various neoplastic processes represents an intensely controversial hypothesis [J]. Rev Med ChirSoc Med Nat Iasi, 2012, 116 (2): 446-451.

[45] Lei Liu, Ma XL, Xiao ZL, et al. Prognostic value of vascular endothelial growth factor expression in resected gastric cancer [J]. Asian Pac J Cancer Prev. 2012; 13 (7): 3089-3097. 
[46] Villarejo-Campos P, Padilla-Valverde D, MartinRM, et al. Serum VEGF and VEGF-C values before surgery and after postoperative treatment in gastric cancer $[\mathrm{J}]$. ClinTransl Oncol, 2013, 15 (4): 265-270.

[47] Bilgiç CI, Tez M. Serum VEGF levels in gastric cancer patients: correlation with clinicopathological parameters [J] Turk J Med Sci. 2015, 45 (1): 112-117.

[48] Wang SD. Changes and significance of serum VEGF, MMP-9 and endostatin in patients with gastric cancer [J]. Shandong Med, 2011, 51 (38): 67-68.
[49] Yao J. Clinical significance of combined detection of IL-6, CA724 and VEGF in patients with gastric cancer [J]. Med Fron, 2018, 8 (31): 141-142.

[50] Liu B, Zhou F, Yang F. bFGF, VEGF and CE were used to predict the efficacy of apatinib in the treatment of advanced gastric cancer [J]. World Latest Med Infor (Electronic Version) 2020, 20 (1): 166-167.

[51] Dong L, Xia M, Shang KX, et al. Changes and clinical significance of serum VEGF, MMP-2 and MMP-9 levels in patients with gastric cancer before and after surgery [J]. J Mol Diag Ther, 2020, 12 (9): 1221-1225. 